\title{
Benefícios do Emprego de Substâncias Funcionais no Desenvolvimento de Produtos
}

\author{
Benefits of Using Functional Substances in Product Development
}

\author{
Régia Saviani Trentin ; Joice Sifuentes dos Santos ${ }^{a}$
}

aUNOPAR, Programa de Pós-Graduação Stricto Sensu em Ciência e Tecnologia de Leite e Derivados. PR, Brasil.

*E-mail: regiatrentin@hotmail.com

\begin{abstract}
Resumo
Com a crescente demanda de consumidores por produtos mais saudáveis, que atuam na prevenção e redução de problemas de saúde, houve um aumento no desenvolvimento de bebidas funcionais. Entre os alimentos funcionais podemos encontrar aqueles beneficiados com probióticos, que são micro-organismos conhecidos como 'bactérias do bem' e prebióticos, que são fibras alimentares que ajudam no crescimento e desenvolvimento das bactérias probióticas. Simbiose é o nome dado a combinação desses dois fatores. O consumo regular de produtos simbióticos acarreta funções benéficas ao organismo humano. Além disso outro produto que pode trazer benefícios ao organismo é o leite de ovelha, que se destaca por sua maior quantidade de minerais, lipídios, proteínas e vitaminas essenciais quando comparado ao leite bovino, outro ponto positivo é a presença do ácido linoleico conjugado que ajuda no controle da redução da gordura corporal, além de diversos peptidios que atuam na ECA (enzima conversora da angiotensina) que por sua vez atua no controle da pressão arterial. O soro de leite também é um produto muito bem visto, pois ao ser empregado enriquece o produto onde foi adicionado, gerando uma diminuição da problemática do excesso de soro na indústria, e tornando-se uma solução viável e lucrativa na fabricação de derivados. Este artigo tem como objetivo trazer os benefícios do emprego do soro de leite de ovelha ultrafiltrado, leite de ovelha, inulina como prebiótico, e Lactobacillus rhamnosus como probiótico no desenvolvimento de uma bebida láctea funcional.
\end{abstract}

Palavras-chave: Leite Ovino. Probióticos. Prebióticos.

\begin{abstract}
With the growing consumer demand for healthier products, which work to prevent and reduce health problems, there has been an increase in the development of functional drinks. Among the functional foods we can find those that benefit from probiotics, which are microorganisms known as 'good bacteria' and prebiotics, which are dietary fibers that help in the growth and development of probiotic bacteria. Symbiosis is the name given to the combination of these two factors. Regular consumption of symbiotic products has beneficial functions for the human organism. In addition, another product that can bring benefits to the body is sheep's milk, which stands out for its greater amount of minerals, lipids, proteins and essential vitamins when compared to bovine milk, another positive point is the presence of conjugated linoleic acid that helps in the control of the reduction of the corporal fat, besides several peptides that act in the ACE (angiotensin converting enzyme) that in turn acts in the control of the arterial pressure. Whey is also a very well-regarded product, because when used it enriches the product where it was added, generating a decrease in the problem of excess whey in the industry, and becoming a viable and profitable solution in the manufacture of derivatives. This article aims to bring the benefits of using ultrafiltered sheep whey, sheep's milk, inulin as a prebiotic, and Lactobacillus rhamnosus as a probiotic in the development of a functional dairy drink.
\end{abstract}

Keywords: Sheep Milk. Probiotics. Prebiotics.

\section{Introdução}

Alimentos funcionais são alimentos que fornecem benefícios fisiológicos, reduzindo o risco de doenças, não sendo destinados para corrigir deficiências, tratar doenças e/ou uso em cuidados paliativos (JONES, 2002). Com a crescente demanda de consumidores por produtos mais saudáveis, que atuam na prevenção e redução de problemas de saúde, houve um aumento no desenvolvimento de bebidas funcionais (BARROS et al., 2019).

Por conta de seus componentes, produtos fermentados podem agregar benefícios à saúde, gerando grande interesse no desenvolvimento desses produtos e contribuindo na demanda por alimentos funcionais (MELINI et al., 2019). De acordo com Badilla et al. (2020), os alimentos fermentados podem trazer contribuições significativas para quem os consomem, além de poderem ser fabricados com técnicas e ingredientes simples e de fácil acesso.

As bebidas prontas para o consumo apresentam potencial para desenvolvimento nos segmentos do mercado, que pode ter sua aplicabilidade como suplementos, por apresentar avanços tecnológicos, mudanças no estilo de vida e utilização de ingredientes como o soro de leite (BALDISSERA et al., 2011).

Segundo Roberfroid (2002), se um alimento demonstrar um conveniente efeito nutricional e ser vantajoso ao organismo do indivíduo que o consome, este pode ser conceituado como um alimento funcional ao consumidor.

Muitos esforços foram empregados para dar aos derivados lácteos propriedades benéficas, como a adição de ingredientes 
de valor agregado, como extratos de plantas prebióticos e probióticos (CHAMPAGNE; DAGA, 2018).

Probióticos são "microorganismos vivos, que quando administrados em quantidades adequadas, conferem benefícios à saúde do hospedeiro" (FAO/WHO, 2001; BRASIL, 2018 HILL et al., 2014).

Já os prebióticos são carboidratos que não são digeríveis, e afetam beneficamente o seu hospedeiro por estimular a produção e atividade de bactérias benéficas no colón, apresentam característica de resistência as enzimas salivares, pancreáticas e intestinais, o que permite sua chegada até o colón (SAAD, 2006).

Outro produto benéfico de possível emprego na produção de bebidas funcionais é o leite de ovelha, que apresenta um grande efeito nas características de constituição e na sua concentração(SKOUFOS et al., 2017). O leite de ovelha possui compostos como proteínas, lactose, sólidos totais, gordura e minerais (MOATSOU; SAKKAS, 2019). Na literatura se encontram várias pesquisas envolvendo as propriedades e os produtos derivado deste leite (WENDORFF; HAENLEIN, 2017).

Este artigo tem como objetivo abordar os beneficios de uma bebida láctea funcional enrequecida com simbióticos, leite e soro de ovelha.

\section{Desenvolvimento}

\subsection{Metodologia}

Este é um estudo de revisão, desenvolvido a partir de publicações científicas disponíveis nas bases de dados do Scielo e ScienceDirect. Livros também serviram como suporte no desenvolvimento do texto.

Palavras chaves como leite, soro de leite, ovelha, prebióticos e probióticos foram empregados para realizar a busca dos artigos pertinentes a pesquisa. Outro método para seleção do material foi por meio da análise dos resumos dos artigos. Fatores como ano e o periódico também foram levados em consideração no momento da escolha. Artigos científicos foram selecionados de 2000 a 2020. Livros foram selecionados em um período mais amplo.

\subsection{Discussão}

\subsubsection{Bebida láctea}

"Entende-se por bebida láctea o produto lácteo resultante da mistura do leite e soro de leite adicionado ou não de produto(s) ou substância(s) alimentícia(s), gordura vegetal, leite(s) fermentado(s), fermentos lácteos selecionados e outros produtos lácteos" (BRASIL, 2005).

Entre os produtos lácteos mais consumidos no mundo, encontram-se as bebidas lácteas fermentadas (CORDEIRO et al., 2019). Além de apresentar um excelente valor nutricional, também podem ser classificadas como alimentos funcionais, de acordo com a sua composição. No Brasil, a Agência Nacional de Vigilância Sanitária (ANVISA) regulamentou os alimentos funcionais pela Resolução RDC $\mathrm{n}^{\circ} 243$, de 26 de julho de 2018, que aprova o regulamento técnico de substâncias bioativas e probióticos isolados com alegação de propriedade funcional ou de saúde (BRASIL, 2018). O consumo destes produtos pode minimizar o risco a algumas doenças e agregar benefícios a saúde de seus consumidores (MACEDO; VÉLEZ-RUÍZ, 2015).

Por ser um produto rico em componentes, como proteínas, minerais e vitaminas, as bebidas lácteas apresentam um importante papel nutritivo a quem os consomem regularmente. É um produto bem aceito por aqueles que buscam alimentos funcionais que contribuam com a boa forma e com dietas saudáveis (THAMER; PENNA, 2006).

As bebidas lácteas são conhecidas em todo mundo e apresentam uma excelente matriz alimentar para a adição de probióticos (TURKMEN et al., 2019). Outra alternativa inovadora é adição de substâncias prebióticas as bebidas, que geram vantagens funcionais a mesma. (THAMER; PENNA, 2006).

\subsubsection{Soro de leite}

Estima-se que no mundo há uma produção de 160 milhões de toneladas de soro de queijo, e que a cada ano esse valor aumenta em 2\% (RAMA et al., 2019). O soro é formado na fabricação de queijo durante a coagulação da caseína do leite. Este subproduto exibe componentes como vitaminas, minerais, proteínas funcionais, lactose, peptídios e lipídios. Esses compostos enriquecem o produto onde o soro for adicionado, gerando uma diminuição da problemática do excesso de soro na indústria, e tornando-se uma solução viável e lucrativa na fabricação de derivados (GUIMARÃES; TEIXEIRA; DOMINGUES, 2010).

Porém, o soro produzido causa poluição ao ambiente, isso se dá ao seu alto valor de conteúdo orgânico, sendo a lactose (75\%) o principal componente poluente (VEERAVALLI; METHEWS, 2018). Por apresentar uma alta demanda bioquímica de oxigênio, cerca de 40 a $60 \mathrm{mil} \mathrm{mg/L}$, e por impossibilitar o seu tratamento nas estações de esgoto normatizada, o descarte de soro de queijo não pode ocorrer, sem seu devido tratamento (KOSSEVA et al., 2009). Este resíduo pode ser até cem vezes mais poluente que o esgoto doméstico, e apresenta alta degradabilidade, que gera grandes danos ambientais, como a destruição e poluição das águas subterrâneas (ANDRADE; ALMEIDA NETO; LOPES, 2015).

Outra prática possível de ser empregada para se fazer a separação do soro dos demais componentes do leite é a tecnologia de ultrafiltração. Esta técnica consiste em separar componentes de um fluido, denominado de permeado ou filtrado, e o soluto de alto peso molecular, que é o retentado ou concentrado. Este processo se dá através da separação por uma membrana semipermeável (PEPPIN; ELLIOT, 2001). Nesta prática, as moléculas de maior peso molecular ficam retidas, 
enquanto as de menor peso molecular passam pelo poro da membrana (ORDÓNEZ, 2005). Os elementos que apresentam baixa massa molar, como a lactose, sais e água permeiam pela membrana, já as proteínas ficam retidas ao retentado (LEITE; VAITSMAN; DUTRA, 2006).

De acordo com Del Colle et al (2011), as formações das membranas podem ser de polímeros orgânicos ou inorgânicos, metal, cerâmica, camadas químicas, líquidos ou gases. As membranas podem apresentar poros e faixa de pressão de tamanhos variados (MULDER, 1996).

Ultrafiltração (UF) é uma técnica muito empregada nas indústrias de laticínios, aplicadas para a concentração e para a extração de sais e lactose, leite e soro (FELLOWS, 2006). Desde de 1981 a UF é uma das técnicas mais empregadas para a recuperação das proteínas solúveis no soro (BRANS et al., 2004).

\subsubsection{Leite de ovelha}

Bebidas lácteas probióticas podem ser produzidas a partir de leite de vaca ou de outras espécies de leite não bovino, como de burras, éguas, ovelhas, camelas e cabras. A propriedade terapêutica desses leites de espécies não bovinas torna-os potencialmente úteis na nutrição humana (RANADHEERA et al 2018).

O leite de ovelha, em comparação ao leite bovino, se destaca por sua maior quantidade de minerais, lipídios, proteínas e vitaminas essenciais. Outro ponto positivo é que o leite de ovelha apresenta maiores concentrações de CLA (PARK et al., 2007). CLA é o ácido linoleico conjugado, possui propriedades benéficas ao organismo como controle das doenças crônicas através da redução da gordura corporal (WANG; JONES, 2004). Por conta de seus compostos bioativos, o ácido linoleico pode ser classificado como uma substância funcional (COSTA et al., 2013). O leite de ovelha é pouco utilizado para se beber, com tudo sua rica composição beneficia o desenvolvimento de produtos derivados deste, como queijos, iogurtes e bebidas lácteas (MOATSOU; SAKKAS 2019). Outro ponto positivo deste produto é que ele apresenta melhor rendimento final (BALTHAZAR et al., 2017).

Em comparação ao leite de vaca, o leite de ovelha possui como característica glóbulos de gordura de tamanhos menores, o que faz com que esse leite possua uma alta digestibilidade. Este benefício se dá por conta de as enzimas digestivas atuarem em uma maior superfície de contato, o que facilita a digestão (MARTINI et al., 2012). Outro fator importante é que o leite de ovelha possui um reduzido número de proteína tipo caseína $\alpha \mathrm{s} 1$, o que torna este leite pouco alergênico, promovendo vantagens à saúde (PARK et al., 2007).

O leite derivado de ovinos apresenta diversos peptídeos que tem como ação a inibição da ECA (enzima conversora da angiotensina). Alguns derivados lácteos, que tem em sua matéria prima o leite de ovelha, dispõe de peptídeos que inibem está enzima, encontrando-se em sua maioria derivada da $\beta$-caseína. Porém, alguns peptídeos derivados da $\kappa$-caseína, que são conseguintes da hidrólise do leite pela quimotripsina, pepsina e tripsina, também executam atividades antioxidante (ALBENZIO et al., 2015). Esta enzima atua no sistema renina-angiotensina-aldosterona, convertendo a angiotensina I, em angiotensina II, que é um vaso constritor. Este fato faz com que a ECA aja no controle da pressão arterial através da regulação de fluidos do corpo (LARAGH; STOERK, 1957).

Devido ao fato de ser um produto rico em nutrientes essenciais, o leite de ovelha oferecer para crianças e adultos um repleto regime nutricional (ALBENZIO et al., 2016).

\subsubsection{Probióticos}

As bactérias produtoras de ácido láctico desempenham tanto importante atribuição na saúde de seus hospedeiros quanto na preservação e produção de alimentos (FERREIRA, 2012). Entre os gêneros de destaque das bactérias ácido lácticas (BAL), encontram-se os Lactobacillus spp. que são microorganismos gram-positivos, não esporulados, não patogênicos, catalase negativa, desprovidos de citocromos, anaeróbios aerotolerantes, ácido-tolerantes e estritamente fermentativos (HOLZAPFEL, 2001).

O grupo Lactobacillus casei é formado pelas espécies $L$. casei, L. paracasei e L. rhamnosus. Essas espécies têm grande utilidade nas indústrias, devido a sua aplicação na fabricação de fermentados e/ou como culturas starters na fabricação de derivados lácteos (FERRERO et al., 1996). Estudos mostram que a utilização prolongada de cepas de Lactobacillus spp. podem influenciar no organismo humano, através da neutralização dos efeitos fisiológicos, e sistema imunológico, além da melhora da microbiota gastrointestinal (DALIU et al., 2019).

Segundo Gomes e Malcata (2008), as fábricas de produtos lácteos são um dos setores que mais desenvolve produtos funcionais que contenham culturas probióticas. Atualmente, no mercado Brasileiro, são encontrados diversos alimentos que contém em sua formulação probióticos, entre esses destacam-se os leites fermentados e iogurtes. Estes produtos são produzidos de preferência com bactérias funcionais, como L. acidophilus, Bifidobacterium spp e L. casei (OLIVEIRA et al., 2002). Como citado, probióticos são microorganismos vivos, que quando administrados em quantidades adequadas, conferem benefícios à saúde do seu hospedeiro (FAO/WHO, 2001; BRASIL, 2018).

O consumo regular de produtos enriquecidos com bactérias probióticas traz funções benéficas ao organismo humano (BURITE; SAAD, 2007). Estes benefícios podem estar ligados a mecanismos alvos do organismo, como intestinos, ajudando nos quadros diarreicos, na intolerância a lactose, no peristaltismo e prevenção de infecções e inflamações, nos rins e fígado inibindo produtos catabólicos, e reduzindo taxas de colesterol, contribuem na absorção de nutrientes, no bem-estar e desenvolvimento dos indivíduos, diminuição de componentes tóxicos, aperfeiçoamento do 
sistema imunológico e urogenital, na prevenção de câncer e osteoporose. Além disso, elevam a biodisponibilidade de nutrientes (GRANATO et al., 2010).

Sabe-se que os lactobacilos produzem exopolissacarídeos (EPS), que por sua vez são classificados como seguros para saúde e vida humana. Os EPS desempenham um papel importante no organismo humano, isso se dá ao fato dos EPS serem polímeros naturais, não tóxicos e biodegradáveis. Esta substância ajuda na resposta imune do corpo humano, e auxilia as bactérias a resistirem ao estresse ambiental (OLEKSY; KLEWICKA, 2018).

Entre as linhagens de L. rhamnosus destaca-se a HN001, que foi isolada de produtos lácteos. Este gênero pode proporcionar efeitos benéficos a seu hospedeiro, como a diminuição da resposta inflamatórias intestinais (BANNA et al., 2017).

\subsubsection{Inulina}

Classificada como polissacarídeo do tipo frutano, a inulina é comumente extraída de raízes de chicória (FRANCK, 2007). E tem se mostrado interessante como substituto de gordura em sobremesas, coberturas, recheios e produtos lácteos (APOLINÁRIO et al., 2014). No trabalho de Borges et al. (2019), a inulina foi utilizada como substituto de gordura em queijo frescal elaborado com leite de ovelha, apresentando bons resultados. Sendo considerada um incremento não calórico, também é empregada como adoçante (COSTA; ROSA, 2006). Além disso, pode influenciar na textura, aeração, cremosidade e viscosidade, mesmo que esteja em baixa quantidade (KIP; MEYER; JELLEMA, 2006).

Um aspecto benéfico da inulina é que sua adição ao produto contribui no aumento da capacidade de sobrevivência de Lactobacillus spp (ÖNAL DARILMAZ et al., 2019), pois a inulina pode ser utilizada pelos Lactobacillus spp como fonte de carbono (BALTHAZAR et al., 2018).

Outros benefícios dessa fibra são diversos, como auxilio na mineralização óssea, controle da glicemia e lipídeos séricos, doença do fígado gorduroso, auxilia na prevenção do câncer de cólon, melhora da imunidade e do metabolismo (KELLY, 2009).

Em um estudo com ratos, a administração de inulina na dieta apresentou uma redução das irregularidades apresentadas no colón quando comparadas a ratos controles (KAUR; GUPTA, 2002). Esse potencial anticarcinogênico ocorre, provavelmente, pela modificação da microbiota do cólon (ROBERFROID, 2002).

A Agência Nacional de Vigilância Sanitária (ANVISA), estipula valores diários de no mínimo $5,0 \mathrm{~g}$ de inulina no alimento, não excedendo $30 \mathrm{~g}$ do produto (BRASIL, 2018).

\section{Conclusão}

A produção de bebidas funcionais vem cada vez mais sendo empregado na indústria. Este produto é muito bem aceito e consumido pelos indivíduos que buscam por uma alimentação mais saudável, rica em nutrientes e proteínas. Com isso, o desenvolvimento de alimentos utilizando leite e soro de ovelha, inulina como prebiótico e Lactobacillus rhamnosus como probiótico, é algo que apresenta um grande potencial de aceitação sensorial e comercial, por conta de seus diversos benefícios, como uma melhor digestibilidade, um melhor controle da pressão arterial, uma melhoria no sistema imune e digestivo daqueles que os consumirem.

\section{Referências}

ALBENZIO, M. et al. Nutritional properties of small ruminant food products and their role on human health. Small Ruminant Res., n.135, p.3-12, 2016. doi: https://doi.org/10.1016/j. smallrumres.2015.12.016

ALBENZIO, M. et al. Identification of peptides in functional Scamorza ovine milk cheese. J. Dairy Scie., n.98, p. 8428-8432, 2015. doi: https://doi.org/10.3168/jds.2015-9844

ANDRADE, R.S.; ALMEIDA NETO, J.A; LOPES, R.C.S.Q. Valorização biotecnológica de soro de leite por fermentação utilizando Saccharomyces cerevisiae. Estudos Tecnol. Eng., v.11, n.2, p 82-91, 2015. doi: 10.4013/ete.2015.112.04

APOLINÁRIO, A.C. et al. Inulin-type fructans: a review on different aspects of biochemical and pharmaceutical technology. Carbohydr. Polym., v.101, n.3, p.368-378, 2014. doi: 10.1016/j. carbpol.2013.09.081

BADILLA, O.S. et al. Use of coconut water (Cocus nucifera L) for the development of a symbiotic functional drink. Heliyon., v.6, p.1-6, 2020. https://doi.org/10.1016/j.heliyon.2020.e03653

BALDISSERA, A. C. et al. Alimentos funcionais: uma nova fronteira para o desenvolvimento de bebidas proteicas a base de soro de leite. Semina: Ciênc. Agrár., v.32, n.4, p.1497-1526, 2011. doi:10.5433/1679-0359.2011

BALTHAZAR, C.F. et al. Sheep milk: physicochemical characteristics and relevance for functional food development. Compr. Rev. Food Sci. Food Saf., v.16, p.247-262, 2017. doi: 10.1111/1541-4337.12250

BALTHAZAR, C.F. et al. The addition of inulin and Lactobacillus casei 01 in sheep milk ice cream. Food Chem., p. 464-472, 2018. doi: 10.1016/j.foodchem.2017.12.002

BANNA G. L. et al. Lactobacillus rhamnosus GG: an overview to explore the rationale of its use in câncer. Front. Pharmacol., v.8, p.603, 2017. doi: 10.3389/fphar.2017.00603

BARROS, C.P. et al. Paraprobióticos: aspectos teóricos e potenciais vantagens da aplicação em produtos lácteos. 2019. Disponível em: https://www.milkpoint.com.br/artigos/industria/ paraprobioticos-aspectos-teoricos-e-potenciais-vantagens-daaplicacao-em-produtos-lacteos-213612/. Acesso em: 18 jul. 2019.

BORGES, J.V. et al. Reduced-fat Frescal sheep milk cheese with inulin: a first report about technological aspects and sensory evaluation. J. Dairy Res., v. 86, p. 368-373, 2019. doi: 10.1017/ S0022029919000487

BRANS, G. et al. Membrane fractionation of milk: state of the art and challenges. J. Membr. Sci., v. 243, n. 2, p.263-272, 2004. doi: 10.1016/j.memsci.2004.06.029

BRASIL. Ministério da Agricultura, pecuária e Abastecimento. Instrução normativa n.16, de 23 de agosto de 2005. Aprova o regulamento técnico de identidade e qualidade de bebida láctea. Brasília: Diário Oficial da República Federativa do Brasil, Poder Executivo, Brasília, DF, 24 ago. 2005. Seção 1, p.7. 
BRASIL. Ministério da saúde. Agência Nacional de Vigilância Sanitária. Relatório de Análise das Contribuições da CP $\mathrm{n}^{\circ}$ 457/2017. Brasília, DF, 2018.

BRASIL. Ministério da saúde. Agência Nacional de Vigilância Sanitária. Resolução RDC nº 243, de 26 de Julho de 2018. Dispõe sobre os requisitos sanitários dos suplementos alimentares. Diário Oficial da União, Brasília, DF, 2018.

BURITI, F. C.A.; SAAD, S. M. I. Bactérias do grupo Lactobacillus casei: caracterização, viabilidade como probióticos em alimentos e sua importância para a saúde humana. Arch. Latinoam. Nutr., v.57, n. 4, p. 373-380, 2007.

CHAMPAGNE, C. P.; DA CRUZ, A. G.; DAGA, M. Strategies to improve the functionality of probiotics in supplements and foods. Curr. Opin. Food Sci., n.22, p.160-166, 2018. doi: 10.1016/j. cofs.2018.04.008

CORDEIRO, M.A.E.L.S. et al. Fermented whey dairy beverage offers protection against Salmonella enterica ssp. enterica serovar Typhimurium infection in mice. J. Dairy Sci, v.102, p.6756-6765, 2019. doi: $10.3168 /$ jds.2019-16340

COSTA, M.P. et al. Leite fermentado: Potencial alimento funcional. Encicl. Biosf., v.9, p.1387-1408, 2013.

DALIU, P., SANTINI, A., NOVELLINO, E. From pharmaceuticals to nutraceuticals: Bridging disease prevention and management. Expert Rev. Clin. Phar, p. 1-7, 2019. doi: $10.1080 / 17512433.2019 .1552135$

DEL COLle, R; FORTUlAN, C. A.; FONTES, S.R. Manufacture and characterization of ultra and microfiltration ceramic membranes by isostatic pressing. Ceram. Int., v.37, n.4, p. 1161-1168, 2011. doi: 10.1016/j.ceramint.2010.11.039

FAO/WHO. Food and Agriculture Organization of United Nations; World Health Organization. Food and Nutrition paper 85. Probiotics in food - Health and nutritional properties and guidelines for evaluation. (2006). Report of a Joint FAO-WHO expert consultation on evaluation of health and nutritional properties of probiotics in food including powder milk with live lactic acid bacteria. Cordoba, Argentina, 2001

FELLOWS, P.J. Tecnologia do processamento de alimentos: princípios e práticas. Porto Alegre: Artmed, 2006.

FERREIRA, C.L.L.F. Grupo de bactérias lácticas e aplicação tecnológica de bactérias probióticas. In: FERREIRA, C.L.L.F. Prebioticos e probióticos: atualização e prospecção. Rio de Janeiro: Rubio, 2012. p.1-28

FERRERO M. et al. Molecular characterization of Lactobacillus casei strains. FEMS Microbiol. Lett., p.215-219, 1996. doi: 10.1111/j.1574-6968.1996.tb08339.x

FRANCK, A. Technological functionality of inulin and oligofructose. Brit. J. Nutrit., v. 87, p. 287-291, 2007. doi: 10.1079 / BJNBJN / 2002550

GOMES, A.M.P.; MALCATA, F.X. Bifidobacterium spp. and Lactobacillus acidophilus: biological, biochemical, technological and therapeutical properties relevant for use as probiotics. Trends Food Sci. Technol., v.10, p.139-157, 2008. doi: 10.1016/S09242244(99)00033-3

GRANATO, D. et al. Probiotic dairy products as functional foods. Compr. Rev. Food Sci. Food Saf., v. 9, n. 5, p. 455-470, 2010. doi: 10.1111/j.1541-4337.2010.00120.x

GUIMARÃES, P.M.R.; TEIXEIRA, J.A.; DOMINGUES, L. Fermentation of lactose to bioethanol by yeasts as part of integrated solutions for the valorization of cheese whey. Biotechnol. Adv., v. 28, n.3, p.375-388, 2010. doi: 10.1016/j.biotechadv.2010.02.002
HILL, C.; GUARNER, F.; REID, G. et al. The International Scientific Association for Probiotics and Prebiotics consensus statement on the scope and appropriate use of the term probiotic. Nat. Rev. Gastroenterol. Hepatol., v. 11, p.506-514, 2014.

HOLZAPFEL, W.H. et al. Taxonomy and important features of probiotic microorganisms in food and nutrition. Am. J. Clin. Nutr., p.365-373, 2001. doi: 10.1093/ajen/73.2.365s

JONES, P.J. Clinical nutrition: 7. Functional foods - more than just nutrition. CMAJ, v.166, n.12, p.1555-1563, 2002.

KAUR, N.; GUPTA, A.K. Applications of inulin and oligofructose in health and nutrition. J. Bioscie. v.27, p.703-714, 2002. doi: 10.1007/BF02708379

KELLY, G. Inulin-type prebiotics: a review (Part 2). Alternative Med. Rev., v.14, n.5, p.36-55, 2009.

KIP, P.; MEYER, D; JELLEMA, R.H. Inulins improve sensoric and textural properties of low-fat yoghurts. Int. Dairy J., v.16, n.9, p.1098-1103, 2006. doi: 10.1016/j.idairyj.2005.10.011

KOSSEVA, M. R. et al. Use of immobilised biocatalysts in the processing of cheese whey. Int. J. Biol. Macromol., v.45, n.5, p.437-447, 2009. doi: 10.1016/j.ijbiomac.2009.09.005

LARAGH, J.H; STOERK, H.C. A Study of the Mechanism of Secretion of the Sodium-Retaining Hormone (Aldosterone). $J$. Clinical Invest., v.36, n.3, p.383/392. doi: 10.1172/JCI103434

LEITE, Z.T.C.; VAITSMAN, D.S.; DUTRA, P.B. Leite e alguns de seus derivados: da antiguidade à atualidade. Quím. Nova, v.29, n.4, p.876-880, 2006. doi: 10.1590/S0100-40422006000400043

MACEDO, R. C.; J. F. VÉLEZ-RUÍZ. Propiedades fisicoquímicas $\mathrm{y}$ de flujo de un yogur asentado enriquecido con microcápsulas que contienen ácidos grasos omega 3. Inf. Tecnol., n.26, p.87-96, 2015. doi: 10.4067/S0718-07642015000500012

MARTINI, M.; ALTOMONTE, I.; SALARI, F. Relationship between the nutritional value of fatty acid profile and the morphometric characteristics of milk fat globules in ewe's milk. Small Ruminant. Res., v.105, p.33-37, 2012. doi: 10.1016/j. smallrumres.2011.12.007

MELINI, F. Health-promoting components in fermented foods: an up-to-date systematic review. Nutrients, v.11 , p.1-24, 2019. doi: 10.3390/nu11051189

MOATSOU, G; SAKKAS, L. Sheep milk components: focus on nutritional advantages and biofunctional potential. Small Ruminant. Res., p.86-99, 2019. doi: 10.1016/j. smallrumres.2019.07.009

MULDER, M. Basic principles of membrane technology. 2a Edição, Kluwer Academic Publishers, Dordrecht, 1996.

OLEKSY, M.; KLEWICKA, E. Exopolysaccharides produced by Lactobacillus sp.: Biosynthesis and applications. Crit. Rev. Food Sci. Nutr., v.58, n.3, p.450-462, 2018. doi:10.1080/10408398.20 16.1187112

OLIVEIRA, M.N. et al. Aspectos Tecnológicos de alimentos funcionais contendo probióticos. Rev. Bras. Ciênc. Farm., v.38, n.1, p.1-21, 2002.

ÖNAL, D; SÖNMEZ, S; BEYATLI, Y. The effects of inulin as a prebiotic supplement and the synbiotic interactions of probiotics to improve oxalate degrading activity. Int. J. Food Sci. Technol., v.54, p.121-131, 2019.

ORDÓÑEZ, J.A. Tecnologia de alimentos: componentes dos alimentos e processos. Porto Alegre: Artmed, 2005.

PARK, Y. W. et al. Hysicochemical characteristics of goat and sheep milk. Small Ruminant. Res., v. 68, p.88-113, 2007. 
doi: 10.1016/j.smallrumres.2006.09.013

PEPPIN, S.L.; ELLIOT, J.A.W. Non-equilibrium thermodynamics of concentration polarization. Adv. Colloid Interface Sci., v.92, n.1-3, p.1-72, 2001. doi: 10.1016/S0001-8686(00)00029-4

RANADHEERA, C.S.; NAUMOVSKI, N.; AJLOUNI, S. Nonbovine milk products as emerging probiotic carriers: recent developments and innovations. Curr. Opin. Food Sci., n.22, p.109-114, 2018. doi: 10.1016/j.cofs.2018.02.010

RAMA, G.R. et al. Potential applications of dairy whey for the production of lactic acid bacteria cultures. Int. Dairy J., v.98, p 25-37, 2019. doi:10.1016/j.idairyj.2019.06.012

ROBERFROID, M. B. Functional foods: concepts and application to inulin and oligofructose. Br. J. Nutr., v. 87, n.2, p.139-143, 2002. doi: 10.1079/BJNBJN/2002529

ROBERFROID, M.B. Functional food concept and its application to prebiotics. Digest. Liver Dis., v. 34, n.2, p.105-10, 2002. doi: 10.1016/S1590-8658(02)80176-1

SAAD, S.M.I. Probióticos e prebióticos: o estado da arte. Braz. J. Pharm. Sci., v.42, n.1, p.1-16, 2006. doi: 10.1590/S151693322006000100002

SKOUFOS, I. et al. Milk quality characteristics of Boutsiko,
Frisarta and Karagouniko sheep breeds reared in the mountainous and semimountainous areas of Western and Central Greece. Int. J. Dairy Technol., n.70, p.345-353, 2017. doi: 10.1111/14710307.12349

THAMER, K. G.; PENNA, A. L. B. Caracterização de bebidas lácteas funcionais fermentadas por probióticos e acrescidas de prebióticos. Ciênc.Tecnol. Alim., v. 26, n.3, p.1-7, 2006. doi: 10.1590/S0101-20612006000300017

TURKMEN, N.; AKAL, C.; OZER, B. Probiotic dairy-based beverages: a review. J. Funct. Foods, v.53, p.62-75, 2019. doi: 10.1016/j.jff.2018.12.004

VEERAVALLI, S.S.; MATHEWS, A.P. Exploitation of acidtolerant microbial species for the utilization of low-cost whey in the production of acetic acid and propylene glycol. Appl. Microbiol. Biotechnol, n.102, p. 8023-8033, 2018. doi: 10.1007/ s00253-018-9174-3

WANG, Y.M.; JONES, P.J.H. Conjugated linoleic acid and obesity control: efficacy and mechanisms. Int. J. Obes., v.28, n.8, p.941-55, 2004. doi: 10.1038/sj.ijo.0802641

WENDORFF, W.L; HAENLEIN, G.F.W. Sheep milkcomposition and nutrition. Handbook of Milk of non-Bovine Mammals. Hoboken: John Wiley Sons, 2017. 